\title{
The Interplay Between Homeostatic Synaptic Plasticity and Functional Dendritic Compartments
}

Ithai Rabinowitch and Idan Segev

J Neurophysiol 96:276-283, 2006. First published Mar 22, 2006; doi:10.1152/jn.00074.2006

You might find this additional information useful...

This article cites 47 articles, 18 of which you can access free at:

http://jn.physiology.org/cgi/content/full/96/1/276\#BIBL

This article has been cited by 1 other HighWire hosted article:

Synaptic Democracy in Active Dendrites

C. C. Rumsey and L. F. Abbott

J Neurophysiol, November 1, 2006; 96 (5): 2307-2318.

[Abstract] [Full Text] [PDF]

Updated information and services including high-resolution figures, can be found at:

http://jn.physiology.org/cgi/content/full/96/1/276

Additional material and information about Journal of Neurophysiology can be found at: http://www.the-aps.org/publications/jn

This information is current as of November 4, 2006. 


\title{
The Interplay Between Homeostatic Synaptic Plasticity and Functional Dendritic Compartments
}

\author{
Ithai Rabinowitch and Idan Segev \\ Interdisciplinary Center for Neural Computation and Department of Neurobiology, Institute for Life Sciences, Hebrew University \\ of Jerusalem, Israel
}

Submitted 23 January 2006; accepted in final form 14 March 2006

\begin{abstract}
Rabinowitch, Ithai and Idan Segev. The interplay between homeostatic synaptic plasticity and functional dendritic compartments. $J$ Neurophysiol 96: 276-283, 2006. First published March 22, 2006; doi:10.1152/jn.00074.2006. Homeostatic synaptic plasticity (HSP) is an important mechanism attributed with the slow regulation of the neuron's activity. Whenever activity is chronically enhanced, HSP weakens the weights of the synapses in the dendrites and vice versa. Because dendritic morphology and its electrical properties partition the dendritic tree into functional compartments, we set out to explore the interplay between HSP and dendritic compartmentalization. For this purpose, we used a detailed model of a CA1 pyramidal neuron receiving a large number of activity-dependent plastic synapses and developed a novel approach for specifying functional dendritic subunits. We found that the degree of dendritic compartmentalization and the location-specificity of HSP are strongly tied. A local HSP mechanism, operating at the level of the individual synapse, will regard the neuron as a multiunit distributed system, each unit consisting of many synapses, and will thus support dendritic compartmentalization, whereas a global HSP mechanism, modifying all synapses in unison, will treat the neuron as a single centralized unit. Both local and global HSP can successfully counterbalance persistent, cell-wide perturbations of dendritic activity. The spatial distribution of synaptic weights throughout the dendrites will markedly differ under the local versus global HSP mechanisms. We suggest an experimental paradigm to unravel which type of HSP mechanism operates in the dendritic tree. The answer to this question will have important implications to our understanding of the functional organization of the neuron.
\end{abstract}

\section{N T R O D U C T I O N}

Homeostatic synaptic plasticity (HSP) is a very slow (hoursto-days time scale) negative feedback process adjusting synaptic weights to apparently compensate for persistent deviations in the level of neuronal activity. Experiments have shown that under HSP, a chronic rise in activity leads to synaptic weakening and vice versa (reviewed in Turrigiano and Nelson 2004). HSP could be associated with other negative-feedback processes that presumably balance synaptic strength (Abbott and Nelson 2000; Burrone and Murthy 2003) such as synaptic depression during spike-timing-dependent plasticity (Song et al. 2000), meta-plasticity (in the sense of a sliding threshold for potentiation/depression) (Abraham and Bear 1996) and antiHebbian forms of plasticity (Rumsey and Abbott 2004). What distinguishes HSP from the others is its very slow dynamics and that it is independent of synaptic activation (Leslie et al. 2001; O’Brien et al. 1998; Rao and Craig 1997; Thiagarajan et

Address for reprint requests and other correspondence: I. Segev, Interdisciplinary Center for Neural Computation and Department of Neurobiology, Institute for Life Sciences, Edmond Safra campus, Givat Ram, Jerusalem 91904, Israel (E-mail: idan@lobster.1s.huji.ac.il). al. 2005; Turrigiano et al. 1998). Thus it seems that HSP will seek to adapt the neuron to persistently perturbed levels of activity, regardless of the source of perturbation.

One important unresolved question regarding HSP in the neuron's dendrites is whether it is local or global. We define local HSP (l-HSP) as fulfilling two conditions. First, the strength of each synapse may be individually modified. Second, the HSP mechanism must be sensitive to the average level of activity at each individual synaptic location. If one or both of these conditions are not satisfied, then HSP is considered to be global ( $g$-HSP). Recent experimental studies have demonstrated individual synaptic modification during HSP (Ju et al. 2004; Sutton et al. 2004), but the spatial sensitivity of the HSP mechanism remains unknown. Furthermore, the effective spatial resolution of HSP is likely to depend not only on its underlying mechanism but also on spatial interactions in the dendritic tree and on the spatiotemporal input pattern. Thus the question arises, what is the interplay between HSP, dendritic structure and the spatial organization of dendritic activity?

To investigate this question, we used a detailed model of a CA1 pyramidal neuron receiving numerous synaptic inputs and incorporated either a $l$-HSP or a $g$-HSP mechanism into these synapses. We compared how each of these two mechanisms interacts with various aspects of the spatial structure of dendritic activity such as the back-propagating action potential (BPAP) that invades large portions of the dendritic tree, cellwide perturbations to activity as are typically induced in experiments on HSP, local perturbations to activity affecting only a subregion of the dendrites, and the natural partitioning of the dendrites into functional compartments (Hausser and Mel 2003; Poirazi et al. 2003; Polsky et al. 2004; Wei et al. 2001; Williams 2004).

To systematically identify and map such dendritic compartments in our model, we developed a novel measure based on the peak cross-correlation of activity between different regions of the dendritic tree. Unlike previous attempts to characterize dendritic compartments (Koch et al. 1982; Zador et al. 1995), the present approach can be used to study dendritic compartmentalization of any sort [e.g., voltage (Hausser and Mel 2003) or ion concentration (Helmchen 1999; Larkum et al. 2001; Zador and Koch 1994)], under any condition (e.g., specific spatiotemporal input patterns, nonlinear dendrites), and can capture cooperative effects and not just isolated pair-wise relations between dendritic sites.

The costs of publication of this article were defrayed in part by the payment of page charges. The article must therefore be hereby marked "advertisement" in accordance with 18 U.S.C. Section 1734 solely to indicate this fact. 
We found that both HSP mechanisms will proportionally scale the strength of synapses on the dendrites of the postsynaptic neuron. However, whereas $g$-HSP can only act globally, scaling all synaptic strengths in unison at the level of the entire neuron, $l$-HSP can change its behavior according to the condition to which it is adapting the neuron. Specifically, $l$-HSP will respond globally, indistinguishably from $g$-HSP, to chronic cell-wide alterations in the level of activity but will selectively scale synaptic weights within individual subregions of the dendrites, such as dendritic compartments, in response to long-lasting perturbations to the activity in these subregions.

\section{METHODS}

\section{Compartmental model}

All simulations were designed and run using NEURON 5.4 (http:// www.neuron.yale.edu). A reconstructed hippocampal CA1 pyramidal cell (Fig. 1A, kindly provided by N. Spruston) equipped with a cylindrical axon was used as an electrically distributed neuron model. Passive properties were as follows: Intracellular resistivity, $R_{\mathrm{a}}=150$ $\Omega \mathrm{cm}$ in dendrites and $50 \Omega \mathrm{cm}$ in the axon; membrane resistance, $R_{\mathrm{m}}=28 \mathrm{k} \Omega \mathrm{cm}^{2}$; membrane capacitance, $C_{\mathrm{m}}=1 \mu \mathrm{F} / \mathrm{cm}^{2}$; resting potential, $V_{\text {rest }}=-65 \mathrm{mV}$. Dendritic spines were accounted for by a twofold increase in $C_{\mathrm{m}}$ and a corresponding decrease in $R_{\mathrm{m}}$. Dendritic sections were divided into discrete $\Delta x=25 \mu \mathrm{m}$ long segments, and the integration time step was $\Delta t=25 \mu \mathrm{s}$ in all simulations.

\section{Voltage-dependent currents}

Voltage-dependent fast $\mathrm{Na}^{+}\left(I_{\mathrm{Na}}\right)$, delayed rectifier $\mathrm{K}^{+}\left(I_{\mathrm{K}}\right)$ and A-type $\mathrm{K}^{+}\left(I_{\mathrm{A}}\right)$ currents as in Migliore et al. (1999) were incorporated in the neuron, with the following minor modifications. Only the proximal variant of $I_{\mathrm{A}}$ was used (Migliore et al. 1999); the additional inactivation gate for $I_{\mathrm{Na}}$ (Migliore 1996) was not employed; and $V_{1 / 2}$ for activation of $I_{\mathrm{Na}}$ in the axon was raised from -30 to $-25 \mathrm{mV}$. $I_{\mathrm{Na}}$, $I_{\mathrm{K}}$, and $I_{\mathrm{A}}$ channel densities in the dendrites were 60,50 , and $100+$ $1.22 \cdot x \mathrm{pS} / \mu \mathrm{m}^{2}$, respectively, where $x$ indicates path distance from the soma in $\mu \mathrm{m}$. Channel densities in the soma were identical to those in the adjoining dendritic branches. $I_{\mathrm{Na}}, I_{\mathrm{K}}$, and $I_{\mathrm{A}}$ channel densities in the axon were $35,000,500$, and $4,000 \mathrm{pS} / \mu \mathrm{m}^{2}$, respectively. In the
"no-AP" condition (in Figs. 1 and 4), no active channels were included in the axon.

\section{Synapses and HSP}

Excitatory synaptic conductance was modeled as a double exponential (the built in NEURON point process Exp2Syn was used) with $\tau_{\text {rise }}=0.3 \mathrm{~ms}, \tau_{\text {decay }}=3 \mathrm{~ms}$, and reversal potential, $E_{\text {rev }}=0$. One such model synapse was included in each one of the 523 dendritic segments and randomly activated according to a Poisson distribution with a mean rate of $2 \mathrm{~Hz}$, unless otherwise mentioned. The time constant for the HSP model, $\tau_{\mathrm{H}}$, (Eqs. 1 and 2) was set to $100 \mathrm{~s}$; larger $\tau_{\mathrm{H}}$ values only increased the simulation time without changing the results.

\section{Simulations}

Four types of simulations were run. 1) No-HSP, in which synaptic conductances were "frozen" either at their initial condition prior to HSP or at HSP steady-state values. These simulations were run for 100-s simulation time. 2) $g$-HSP, which modified synapses according to Eq. 1, were run for 200- to 400-s simulation time. 3) $l$-HSP, which modified synapses according to Eq. 2, were run for 500- to 1,500-S. 4) EPSC, in which the built in NEURON voltage-clamp model, VClamp, was inserted in the soma, and its current was recorded for each individually activated synapse for 50 -ms simulation time.

\section{Novel method for quantifying dendritic compartmentalization}

Cross-correlations $\left(\mathrm{CC}_{i, j}\right)$ at $-50<\Delta \tau<50$-ms time delays between every two dendritic sites, $i$ and $j$, were computed over 5-s-long voltage traces recorded simultaneously at all sites in no-HSP simulations with AP generation blocked and a peak CC (PCC) matrix was produced. The following steps were then carried out to identify the sites that did not belong to any compartment (the "link" subregions, where activity is similar within but also similar to that of the compartments connected to it, e.g., the main apical trunk).

First, two thresholds for the PCC between every two sites were defined: a high-threshold, $\theta_{\mathrm{h}}=0.45$, above which sites were considered to be similar; and a low threshold, $\theta_{1}=0.25$, below which sites were considered to be dissimilar. These values were chosen from
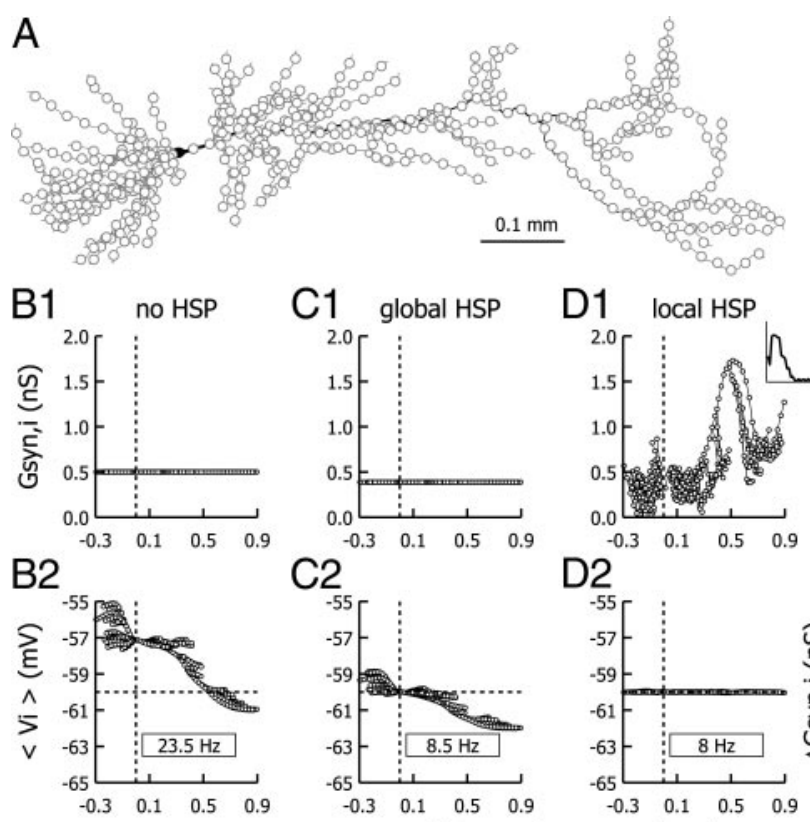
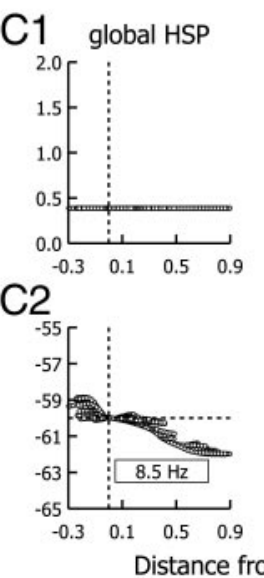

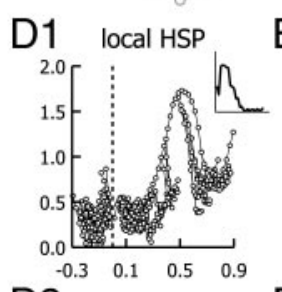

\section{D2}

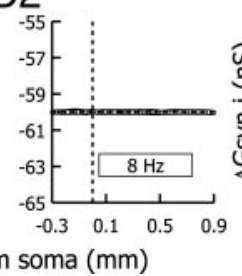

E1
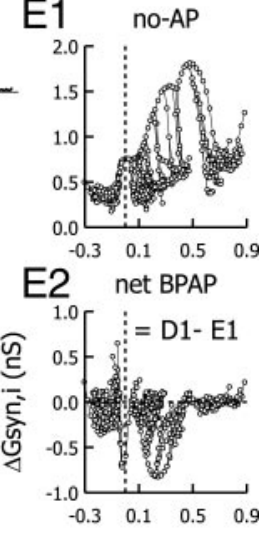

FIG. 1. Shaping synaptic weight distribution with $l$-HSP vs. g-HSP. A: compartmental model of a CA1 pyramidal neuron (kindly provided by N. Spruston) receiving 523 excitatory synaptic contacts $(\mathrm{O})$. Synapses were randomly activated at an average rate of $2 \mathrm{~Hz}$. B1-D1: synaptic conductance, $G_{\mathrm{syn}, i}$, plotted for each synaptic site, $i$, against its distance from the soma prior to HSP $(B 1)$, following $g$-HSP $(C l)$ and $l$-HSP (D1). Inset in D1: the $G_{\mathrm{syn}, i}$ histogram (abscissa, 0-2 nS; ordinate: $0-15 \%$ ). $B 2-D 2$ : corresponding time-averaged membrane potential, $\left\langle V_{i}\right\rangle$, at each site, $i$ and average AP rate (box). $---, V_{\mathrm{trg}}=-60 \mathrm{mV} . E 1: G_{\mathrm{syn}, i}$ distribution following $l$-HSP in the absence of APs. E2: net effect of back-propagating action potentials (BPAPs) on $l$-HSP presented as the difference between control (DI) and "no-AP" (E1). Vertical - -, soma location. 
representative sites that behaved like separate compartments, such as in Fig. 4C. Second, for each site, $i$, a list of similar sites, $L_{i}$ (with $\mathrm{PCC}_{i, j}>\theta_{\mathrm{h}}, \mathrm{j} \in L_{i}$ ), was compiled. Third, for each site in the list, $j$, a sub-list, $\mathrm{SL}_{i, j}$, of similar sites (with $\mathrm{PCC}_{j, k}>\theta_{1}, k \in \mathrm{SL}_{i j}$ ) was compiled. Fourth, the intersection of all the sub-lists $I_{i}=\bigcap_{j} \mathrm{SL}_{i, j}$ was computed and compared with the original list, $L_{i}$. Fifth, if not all the sites in the list appear in the intersection of the sub-lists $\left(L_{i} \not \subset I_{i}\right)$, then the site under consideration is similar to at least two sites, $j$ and $k$ $\left(\mathrm{PCC}_{i, j}>\theta_{\mathrm{h}} ; \mathrm{PCC}_{i, k}>\theta_{\mathrm{h}}\right)$, that are dissimilar one to the other $\left(\mathrm{PCC}_{j, k}<\theta_{1}\right.$ ) and thus are not supposed to be in the same compartment. In such a case, the site under consideration, $i$, is marked as a noncompartment (or a "link") site.

Following this analysis, the remaining sites were clustered into compartments using the Matlab built-in hierarchical clustering algorithm, where distance between every two sites, $i$ and $j$, was defined as $1-\mathrm{PCC}_{i j}$, a nearest neighbor measure was used for the linking stage and an inconsistency cutoff value of 0.9 was used as the criterion for clustering links together. Alternatively, any standard clustering algorithm could be used for this stage.

\section{RES ULTS}

\section{l-HSP and g-HSP in dendrites}

We formulated dynamic equations for both $l$-HSP and $g$ HSP and incorporated them in a detailed model of a CA1 pyramidal neuron (Fig. 1A) with numerous excitatory synapses randomly activated throughout the dendrites $(\bigcirc)$. For HSP to occur, a persistent change in average membrane potential is sufficient (Burrone et al. 2002; Leslie et al. 2001; Molder et al. 2004; Paradis et al. 2001). Therefore we assumed that HSP controls the average membrane potential, $\left\langle V_{i}\right\rangle$, either directly or indirectly (e.g., through calcium concentration).

In the model, peak synaptic conductance, $G_{\mathrm{syn}, i}$ at each location $i$, was multiplied by a scaling factor, $\mathrm{SF}_{i}$, reflecting individual modification of synaptic strength ( $\mathrm{Ju}$ et al. 2004; Sutton et al. 2004). $\mathrm{SF}_{i}$ changed according to the difference between the actual membrane potential and a target voltage, $V_{\text {trg }}$. For $g$-HSP, with only cell-wide sensitivity to the level of activity, we chose the somatic membrane potential, $V_{\text {soma, }}$ as the global variable being controlled

$$
\tau_{\mathrm{H}} \cdot \frac{\mathrm{dSF}_{\mathrm{i}}}{\mathrm{d} t}=\frac{V_{\text {trg }}-V_{\text {soma }}}{\kappa} ; \quad \text { if } \mathrm{SF}_{\mathrm{i}}<0 \Rightarrow \mathrm{SF}_{\mathrm{i}}=0
$$

where $\tau_{\mathrm{H}}$, the time constant for the HSP process, is larger by several orders of magnitude than the membrane time constant, and $\kappa=1$, in units of $\mathrm{mV}$, maintains $\mathrm{SF}_{i}$ a unit-less variable. An alternative global variable we tried was the average membrane potential over all $N$ synaptic sites, $1 / \mathrm{N} \cdot \sum_{i} V_{i}(t)$, with no significant impact on our conclusions.

For $l$-HSP, each $S F_{i}$ was modified according to the local membrane potential, $V_{i}$, at each synaptic location $i$, reflecting local sensitivity to the level of activity

$$
\tau_{\mathrm{H}} \cdot \frac{\mathrm{dSF}_{\mathrm{i}}}{\mathrm{d} t}=\frac{V_{\mathrm{trg}}-V_{\mathrm{i}}}{\kappa} ; \quad \text { if } \mathrm{SF}_{\mathrm{i}}<0 \Rightarrow \mathrm{SF}_{\mathrm{i}}=0
$$

Initially, $G_{\mathrm{syn}, i}=0.5 \mathrm{nS}$ for all synapses (Fig. 1Bl). In spite of the uniform synaptic input, the corresponding $\left\langle V_{i}\right\rangle$ had a rather nonuniform shape, covering a range of $\sim 6 \mathrm{mV}$ and an average output firing rate of $23.5 \mathrm{~Hz}$ (Fig. 1B2). This nonuniformity was due to both the varying input resistance along the dendrites and to the nonuniform spatial distribution of active ion channels. We chose $V_{\text {trg }}=-60 \mathrm{mV}$ (between the resting membrane potential and the threshold for AP generation in the model; horizontal dashed lines in Fig. $1 B 2, C 2$, and D2).

$g$-HSP uniformly weakened all synaptic weights by $\sim 25 \%$ (Fig. $1 C 1$ ), successfully bringing $\left\langle V_{\text {soma }}\right\rangle$ to $V_{\text {trg }}$ (Fig. $1 C 2$, intersecting dashed lines) and decreased the AP firing rate (from 23.5 to $8.5 \mathrm{~Hz}$ ). $\left\langle V_{i}\right\rangle$ still covered a range of $\sim 4 \mathrm{mV}$. In contrast, $l$-HSP leveled all $\left\langle V_{i}\right\rangle$ values to $V_{\text {trg }}$ (Fig. 1D2), reducing the average firing rate to $8 \mathrm{~Hz}$. This was achieved by substantially changing the spatial distribution of synaptic weights over the dendritic tree (Fig. 1Dl). This highly nonuniform $G_{\mathrm{syn}, i}$ profile was required to counterbalance the initially nonuniform distribution of $\left\langle V_{i}\right\rangle$ (Fig. 1B2). The shape of the $G_{\mathrm{syn}, i}$ profile is determined by a combination of factors such as the varying input impedance along the dendritic branches resulting in a general decrease in $G_{\mathrm{syn}, i}$ along each branch, dendritic morphology (e.g., denser branching near the soma, entailing lower $G_{\mathrm{syn}, i}$ values in proximity to the soma), the distribution of voltage-dependent ion channels (e.g., an increase in A-type $\mathrm{K}^{+}$channels with distance from the soma, causing a steep increase in $G_{\mathrm{syn}, i}$ along the main apical trunk), the back-propagation of action potentials into the dendrites (compare Fig. 1, DI and El) and the spatial pattern of synaptic activation (see following text). Any changes in these properties will induce a change in the $G_{\mathrm{syn}, i}$ profile. It is noteworthy that for CA1 dendrites, the strength of the Schaffer collateral synapses has been shown to increase with distance along the main apical trunk (Magee and Cook 2000). This could be attributed to a local HSP mechanism (Fig. 1DI). Note also, that the histogram of synaptic weights (Fig. 1D1, inset) was unimodal with a positive skew, in agreement with experimental data (O'Brien et al. 1998) and theoretical considerations (van Rossum et al. 2000).

It is often assumed that HSP is driven by the AP firing rate (Toyoizumi et al. 2005; van Rossum et al. 2000), implying that $l$-HSP will, in effect, show global behavior through the presumably global influence of the BPAP. However, APs are not necessary for HSP to occur in the first place (Ju et al. 2004; Leslie et al. 2001; Sutton et al. 2004), and BPAPs actually have a nonuniform effect on dendritic activity (Goldberg et al. 2002; Lisman and Spruston 2005). For instance, in CA1 pyramidal neurons BPAPs attenuate due to a distance-dependent increase in A-type conductance (Johnston et al. 1999), they do not reach all branches of the dendrite (Golding et al. 2001), and they may well be confounded (Goldberg et al. 2002; Lisman and Spruston 2005) with local dendritic spikes (Ariav et al. 2003; Golding and Spruston 1998). We repeated the $l$-HSP simulation with AP generation blocked and obtained an altered $G_{\text {syn }, i}$ profile (Fig. 1E1, no-AP), which was equally successful in bringing all $\left\langle V_{i}\right\rangle$ values to their target (not shown). The net effect of the BPAPs showed a highly nonuniform contribution of the BPAPs to HSP (Fig. 1E2), implying that even if $l$-HSP was selectively sensitive to BPAPs, it would still act locally.

In summary, both $l$-HSP and $g$-HSP can control the output firing rate of the neuron to approximately the same degree. However, $l$-HSP has direct influence on the spatial distribution of synaptic strength and can regulate the level of activity (e.g., the local value of $\left\langle V_{i}\right\rangle$ ) throughout the dendrites (Fig. 1D2). On the other hand, $g$-HSP has no influence on the shape, but only on the overall magnitude of the spatial distribution of synaptic strength. 


\section{Overall perturbation of activity}

Diffuse perturbation of neuronal activity, induced, for example, by an overall change in the activation of synapses, could occur at times of enhanced or reduced network activity. If this persists for a prolonged period of time, it can be interpreted as a new baseline (set-point) to which neurons in the network should adapt. How does HSP implement this adaptation to persisting changes in the individual neuron's overall activity?

This is actually what all reported experiments on HSP have tested so far (e.g., O'Brien et al. 1998; Rao and Craig 1997; Turrigiano et al. 1998; but see Harms et al. 2005). In all of these experiments, neurons had been subjected to a diffuse and cell- or network- encompassing chronic (several days long) manipulation. This has also been the focus of theoretical work on HSP (Toyoizumi et al. 2005; van Rossum et al. 2000; Yeung et al. 2004), which has assumed a single-compartment neuron where any change in activity is, by definition, a cellwide change. Is there a difference between the responses of $l$-HSP and $g$-HSP to overall changes in activity in a spatially distributed dendritic tree?

To induce such an overall change, and replicate the experimental paradigm, we uniformly altered in Fig. 2 the average rate of synaptic activation from $2 \mathrm{~Hz}$ ("control") to $4 \mathrm{~Hz}$ ("enhanced," red) or to $1 \mathrm{~Hz}$ ("reduced," green) with an initial control distribution (Fig. 2E) as in Fig. 1D1. Sample raster
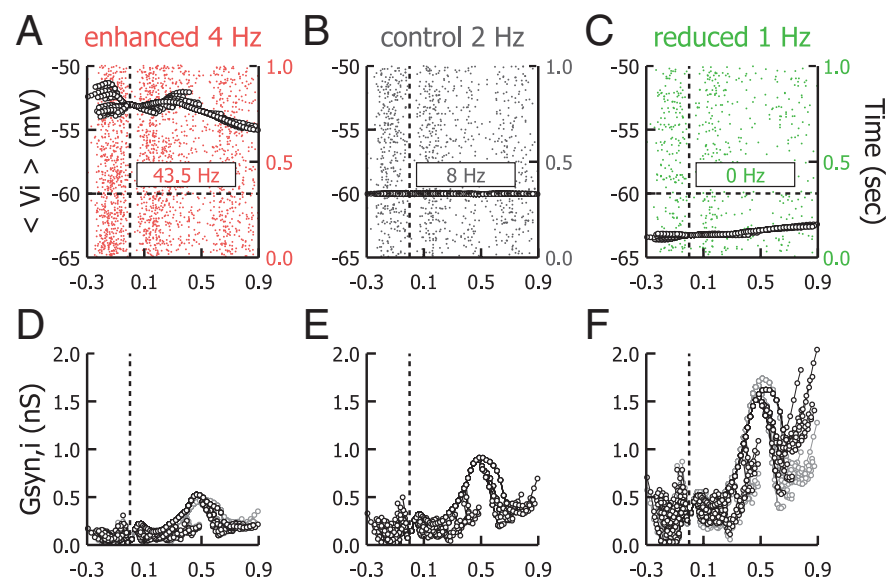

E

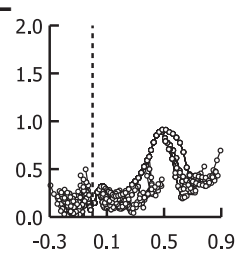

F

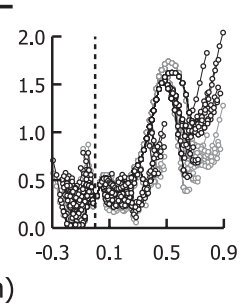

Distance from soma ( $\mathrm{mm})$

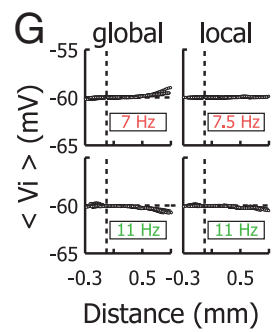$$
\text { o }
$$

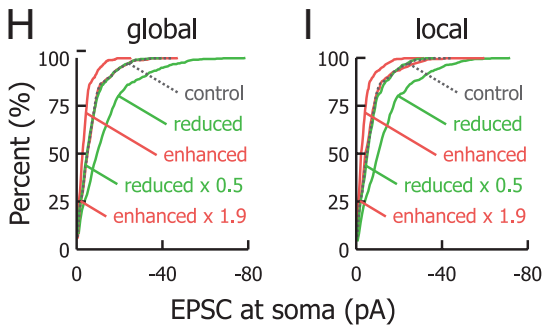

FIG. 2. Both $l$-HSP and $g$-HSP behave globally after overall perturbation of dendritic activity. $A-C:\left\langle V_{i}\right\rangle$ and AP rate (boxes) prior to HSP, superimposed on sample raster plots of synaptic activation times for the enhanced (4 Hz; red), control ( $2 \mathrm{~Hz}$; black), and reduced ( $1 \mathrm{~Hz}$; green) conditions. $E$ : control $G_{\mathrm{syn}, i}$ distribution, as in Fig. 1D1. $D$ and $F: G_{\mathrm{syn}, i}$ distribution following $l$-HSP (dark) and $g$-HSP (light) for the enhanced $(D)$ and reduced $(F)$ activation rates. $G:\left\langle V_{i}\right\rangle$ and AP firing rates following $g$-HSP and $l$-HSP for the enhanced (red, top row) and reduced (green, bottom row) conditions. $H$ and $I$ : cumulative histograms of individual EPSC amplitudes following $g$-HSP and $l$-HSP for each condition and scaled curves fit to the control histogram. plots of synaptic activation times are presented in Fig. 2, $A-C$, on which the corresponding $\left\langle V_{i}\right\rangle$ profiles and average AP firing rates (boxes) prior to HSP are superimposed. The altered level of synaptic activation had a clear impact on $\left\langle V_{i}\right\rangle$ and on the AP rate. The adapted $G_{\mathrm{syn}, i}$ distributions for the enhanced and reduced conditions following HSP are shown in Fig. 2, D and $F$, respectively. The $l$-HSP (dark) and $g$-HSP (light) profiles almost overlapped. The resulting $\left\langle V_{i}\right\rangle$ profiles and AP rates were almost completely restored (Fig. $2 G$ ). The slight deviations of AP rates $(7-7.5 \mathrm{~Hz}$ for the enhanced case and $11 \mathrm{~Hz}$ for the reduced case) from control $(8 \mathrm{~Hz})$ stem from the fact that the firing rate is determined not only by $\left\langle V_{i}\right\rangle$, which is under direct control of the homeostatic process, but also by the extent of membrane potential fluctuations. It is expected theoretically (Abeles 1991) that these fluctuations increase with the square root of input frequency and linearly with excitatory postsynaptic potential (EPSP) amplitude. Therefore a reduction in the input frequency followed by a corresponding increase in EPSP amplitude (as in the reduced case where $G_{\mathrm{syn}, i}$ increased to maintain $\left\langle V_{i}\right\rangle$ constant) is expected to result in increased membrane voltage fluctuations and in consequence, an increase in firing rate and vice versa.

The effect of HSP is often quantified by comparing miniature excitatory postsynaptic current (mEPSC) amplitudes recorded at the soma between treated and control neurons. Cumulative histograms of the mEPSC amplitudes show a uniform multiplicative relationship between the treated and control curves (Desai et al. 2002; Kilman et al. 2002; Turrigiano et al. 1998). Repeating this procedure we found that both $g$-HSP (Fig. $2 H$ ) and $l$-HSP (Fig. $2 I$ ) models fully agree with these experimental results. In both cases, the cumulative histograms of EPSC amplitudes were multiplied by a fixed factor following an overall change in synaptic activation frequency. Thus both $l$-HSP and $g$-HSP can successfully counterbalance persistent, cell-wide perturbations of dendritic activity. Under such conditions they cannot be distinguished.

\section{Selective manipulation of activity}

To distinguish experimentally between $l$-HSP and $g$-HSP, a new experimental design must be applied in which chronic manipulation of activity should vary at different dendritic locations. One possibility would be to expose just a subregion of the dendrites to altered levels of activity for a prolonged period of time. We demonstrate this in our model, by selectively reducing the average synaptic activation rate from 2 to $0.5 \mathrm{~Hz}$ in only a subgroup comprising $\sim 6 \%$ of all synapses (Fig. 3A, dark circles on proximal oblique branches). Note that at the short timescale, a correlation-based ("Hebbian") mechanism could respond to such a selective drop in the level of activity with long-term depression (LTD). In contrast, synaptic strength is expected to increase under $l$-HSP.

$g$-HSP increased the strength of all synapses by $\sim 7 \%$ in compensation for this local reduction in synaptic activation rate without changing the spatial profile of synaptic strength (compare Fig. $3 B$ to Fig. $1 D 1$ ). In contrast, $l$-HSP modified only the affected subregion by selectively strengthening its synapses (Fig. 3C, dark circles). This local response could be discerned in the cumulative EPSC histograms (Fig. 3D), and thus could be considered, if it appeared in an experiment, as evidence for the existence of a $l$-HSP mechanism. Both $g$-HSP and $l$-HSP 


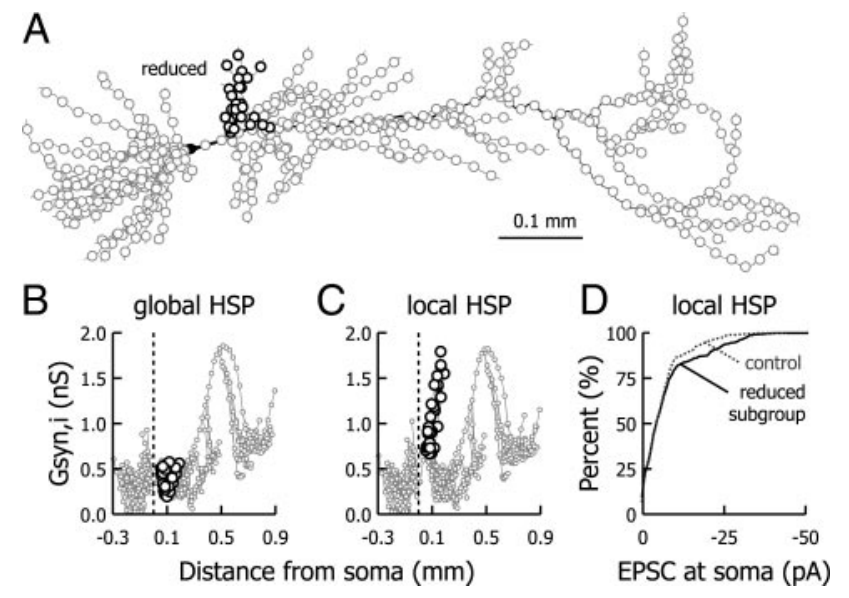

FIG. 3. Suggested experimental design for identifying local HSP. A: selectively "reduced" subgroup consisting of $\sim 6 \%$ of all synapses (dark circles) activated at an average rate of $0.5 \mathrm{~Hz}$, while the rest of the synapses were activated at the baseline average rate of $2 \mathrm{~Hz} . B$ and $C: G_{\mathrm{syn}, i}$ distribution following global $(C)$ and local $(D)$ HSP. Enlarged dark circles represent synapses belonging to the reduced subgroup. $D$ : cumulative histograms of excitatory postsynaptic current (EPSC) amplitudes for control (dotted line) and for local HSP in the reduced subgroup condition (continuous line).

restored $\left\langle V_{\text {soma }}\right\rangle$ and $\left\langle V_{i}\right\rangle$ values to $V_{\text {trg }}$, respectively (not shown).

\section{Functional dendritic compartments}

It appears that between the level of the single synapse and the entire cell, the neuron has an important intermediate level of organization, consisting of numerous individual functional compartments, where "private" interactions can take place (Hausser and Mel 2003; Koch et al. 1982; Polsky et al. 2004; Wei et al. 2001; Williams 2004). The notion of "dendritic compartments" is often used in the literature, but it has never been formally defined and quantified for naturally ongoing in-vivo-like activity. We define dendritic compartments as dendritic subregions within which activity tends to be similar and, at the same time, distinct from activity taking place in other compartments. It must be noted that the compartmentalization is dynamic because it depends not only on the cable properties of dendrites but also on input conditions (including excitability modulation) experienced by the dendritic tree.

We developed a novel procedure for identifying dendritic compartments based on the cross-correlations (CCs) between membrane potential traces (or any other measure of activity) at different dendritic sites. Using especially tailored clustering methods (see METHODS), we delineated the functional compartments in our model neuron (Fig. 4A, arbitrarily colored branches) as well as connecting subregions that did not satisfy the above definition and were therefore classified as "links" between compartments (gray regions). In this example, all synapses were activated randomly with uniform $G_{\mathrm{syn}, i}$ (as in Fig. $1 B 1$ ) and APs were blocked. Thus the compartmentalization obtained by this analysis reveals the inherent electrical partition of the dendritic tree, which could change considerably with the introduction of structure to the input as well as the presence of dendritic spikes and BPAPs.

Sample membrane potential traces recorded simultaneously from four identified representative compartments (recording sites indicated by schematic electrodes) as well as from a link region on the main apical trunk are shown in Fig. $4 B$ (thick overlying traces are for the no-AP condition). The temporal pattern of activity shows salient differences between different compartments (Fig. $4 B$ ) and at the same time, high similarity within each compartment (insets in Fig. 4B), except for occasional variability during synaptic activation. On the other hand, voltage transients in the link trunk region show similarity to transients recorded in the two separate oblique sites (Fig. 4B), although activity between these two compartments is dissimilar. These observations can be quantified by cross-correlating membrane potential traces recorded at different sites, as demonstrated in Fig. 4C. The high 23.5-Hz AP firing rate of the cell introduced large variance in the membrane potential traces, thus dominating the cross-correlation measure and masking the similarities and differences within and between dendritic compartments (thin lines). In realistic, in vivo conditions, the dendritic membrane potential is expected to be much more dominated by synaptic potentials than by the BPAPs (a substantially larger number of synapses producing a considerably higher rate of events relative to somatic AP frequency). Indeed, when APs were blocked, the low cross-correlation values revealed the dissimilarity between the basal 1 and basal2 sites and between the oblique 1 and oblique 2 sites but the high similarity between the oblique 1 and trunk sites (Fig. 4C).

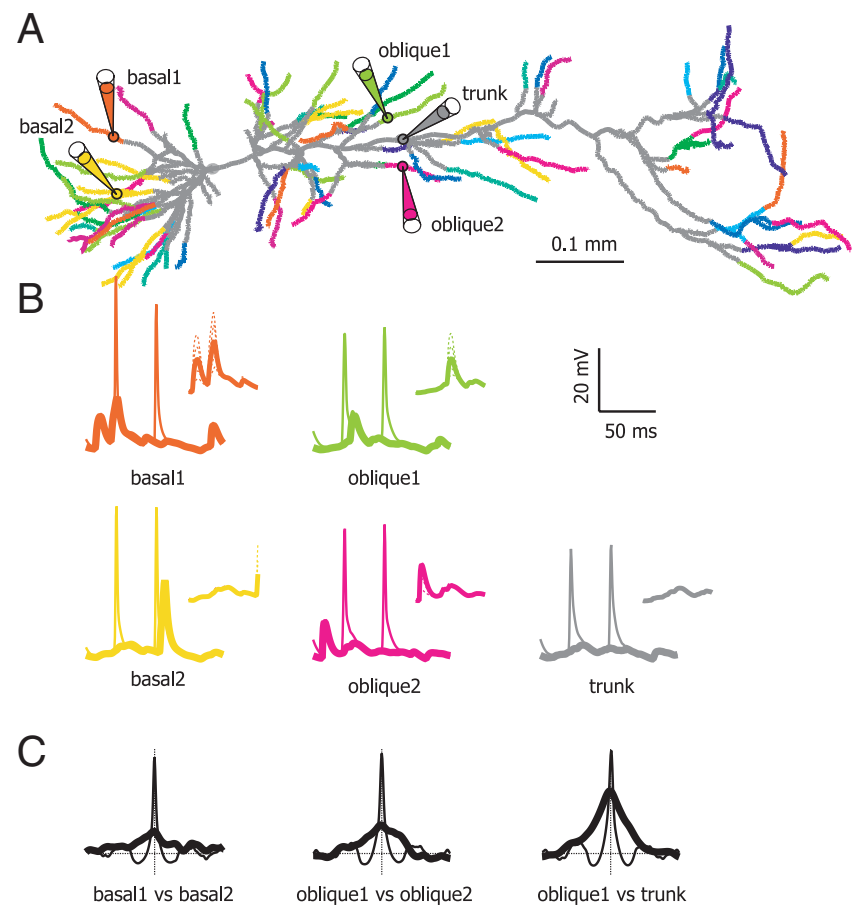

FIG. 4. Dendritic compartmentalization revealed by a novel measure. A: partitioning of the modeled dendritic tree into functional compartments (arbitrary colors) and "links" (gray). Schematic electrodes indicate recording sites at 4 representative compartments and a "link" (the apical trunk). Synapses were randomly activated at $2 \mathrm{~Hz}$ with uniform $G_{\mathrm{syn}, i}=0.5 \mathrm{nS}$. $B$ : sample voltage traces recorded simultaneously for a short epoch from the 5 sites for control (thin lines) and no-AP (thick lines) conditions. For each site, insets show voltage traces recorded from adjacent sites (dotted lines) for the no-AP condition. $C$ : cross-correlation (CC) plots computed between 5-s-long voltage traces recorded from 3 pairs of the representative sites for control (thin line) and no-AP (thick line). Abscissa ranged $\pm 50 \mathrm{~ms}$ (same scale as voltage traces); ordinate ranged $-0.2-1$. 


\section{Dendritic compartments and homeostatic synaptic plasticity}

Can HSP adapt each dendritic compartment autonomously? With its very low spatial resolution, $g$-HSP cannot discern individual compartments and therefore would be incapable of accommodating altered levels of activity within individual compartments. On the face of it, the opposite problem will be presented by $l$-HSP having too high a spatial resolution, adapting each synaptic site individually and not as part of the compartment it belongs to. However, as we have seen in the preceding text, membrane potential varies little within a given compartment (Fig. $4 B$, insets); therefore $l$-HSP is, in effect, expected to modify synapses within the same compartment in unison and thus adapt each compartment individually.

To demonstrate this, we started with the steady-state condition shown in Fig. $1 D 1$ and $D 2$ (where $\left\langle V_{i}\right\rangle=V_{\text {trg }}$ at all sites) and introduced a combination of perturbations to the activity in the model neuron, consisting of an overall decrease in the average synaptic frequency from 2 to $1.5 \mathrm{~Hz}$ and the following increases in activity at the level of individual compartments (Fig. 5). First, the weights of synapses in the basal1 and oblique 1 compartments were randomly potentiated, simulating short timescale plasticity (e.g., long-term potentiation, LTP) within these compartments (Fig. 5Al; enlarged open circles). Second, the average activation rate of the synapses onto the oblique 2 compartment was elevated to a level of $4 \mathrm{~Hz}$, simu-
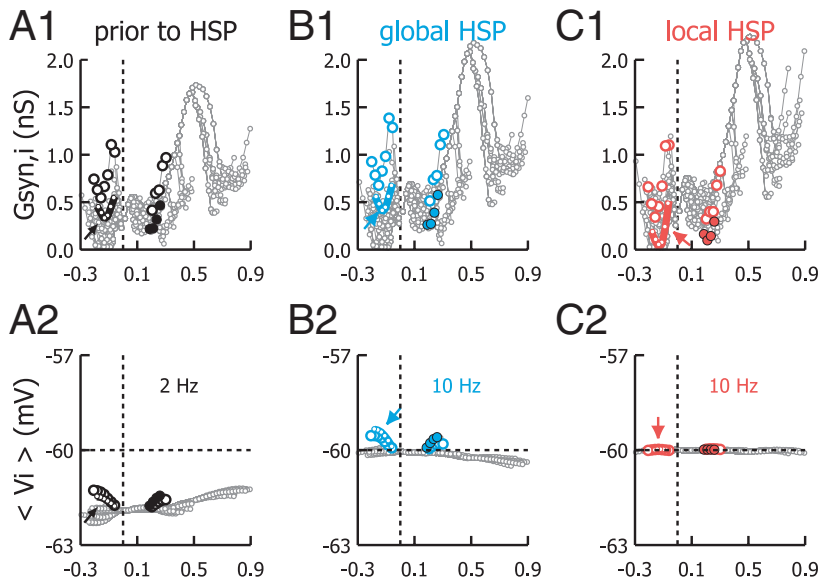

B2
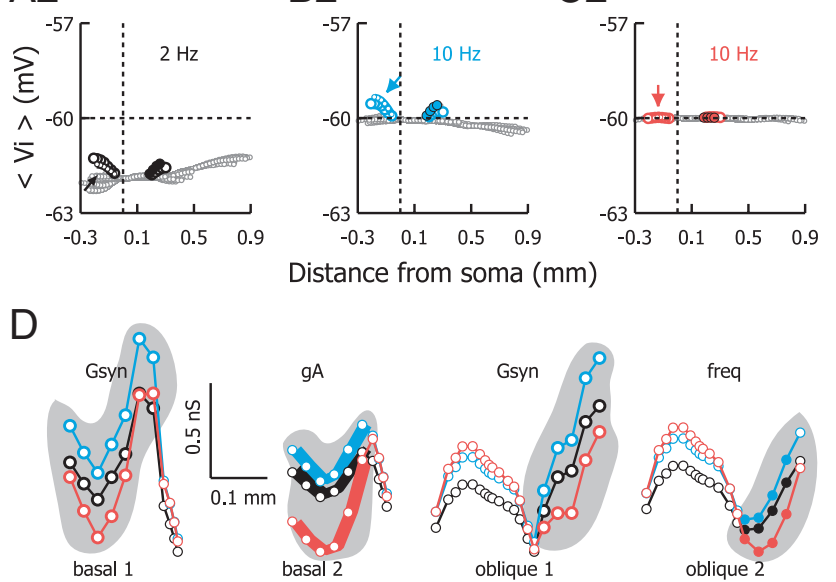

FIG. 5. $l$-HSP vs. $g$-HSP and functional dendritic compartments. Combined perturbations were introduced to the overall activity (reduced input frequency from 2 to $1.5 \mathrm{~Hz}$ ) and to activity in individual compartments (random potentiation of synapses in the basal 1 and oblique 1 compartments; reduced A-type current in the basal2 compartment and enhanced input frequency to 4 $\mathrm{Hz}$ in the oblique 2 compartment). $A-C: G_{\mathrm{syn}, i},\left\langle V_{i}\right\rangle$ and average AP rates (boxes) prior to $\operatorname{HSP}(A, 1$ and 2 , black), following $g$-HSP (B, 1 and 2, blue), and following $l$-HSP $\left(C, 1\right.$ and 2 , red). $D$ : close-ups of the $G_{\mathrm{syn}, i}$ profiles for the perturbed compartments (shaded) prior to HSP (black), following $g$-HSP (blue), and following $l$-HSP (red). Enlarged open circles, potentiated synapses (Gsyn); filled circles, synapses with enhanced activation rate (freq); thickened lines (as well as arrows in $A-C$ ), branch with elevated A-type conductance density (gA). lating a local increase in activity (Fig. 5Al; filled circles). And third, the A-type conductance in the basal2 compartment was reduced, simulating plasticity of dendritic excitability (Frick et al. 2004) (Fig. 5A1; arrow). This selectively amplified the size of the BPAPs in this compartment. The combination of an overall perturbation and perturbations at the level of individual dendritic compartments had a clear effect on the $\left\langle V_{i}\right\rangle$ profile, which dropped from its initial uniform $V_{\text {trg }}$ level (dashed horizontal line in Fig. 5A2) and showed protruding branches resulting from the perturbations of the representative compartments (Fig. 5A2). Concomitantly, the average AP frequency decreased from its $8-\mathrm{Hz}$ baseline to $2 \mathrm{~Hz}$ (Fig. 5A2, box).

$g$-HSP responded to these perturbations by uniformly scaling-up all synaptic weights (compare Fig. 5, Bl and Al), restoring $\left\langle V_{\text {soma }}\right\rangle$ and $\left\langle V_{i}\right\rangle$ at many sites to $V_{\text {trg }}$, as well as the AP rate to $10 \mathrm{~Hz}$ (Fig. 5B2) as compared with $8 \mathrm{~Hz}$ before the perturbation. However, not only was it not capable of adapting the perturbed compartments to the local altered level of activity, it even intensified these perturbations, as can be seen in enlargements of the $G_{\mathrm{syn}, i}$ profile for these compartments (Fig. $5 D$, shaded regions; compare black - prior to HSP, and bluefollowing $g$-HSP). $l$-HSP like $g$-HSP, scaled-up most synaptic weights, counterbalancing the overall decrease in activity (compare Fig. 5, Cl and Al; and Fig. 5D, blue- $g$-HSP, and red-l-HSP, outside the shaded regions). However, at the same time, it also scaled down synaptic weights within each individual perturbed compartment (Fig. 5D; compare black and red), restoring all $\left\langle V_{i}\right\rangle$ values to $V_{\operatorname{trg}}$ (Fig. 5C2). Although these compartments were identified in Fig. 4 under the condition of blocked APs, $l$-HSP was able to respond to the perturbations introduced to these compartments even in the presence of BPAPs because the change in membrane potential within the perturbed compartments was more prominent than the voltage change produced by the BPAPs.

\section{I S C U S S I O N}

To date, homeostatic synaptic plasticity has been studied, to a very large extent, under the conceptual framework of a single compartment ("point-neuron"). In all experiments on HSP, we are aware of, neurons were exposed to chronic altered levels of activity uniformly affecting the entire cell (e.g., O'Brien et al. 1998; Rao and Craig 1997; Turrigiano et al. 1998); but see (Harms et al. 2005). Such overall manipulation, in effect, treats the cell as a single functional compartment. Recently, attempts were made to directly explore the effects of overall manipulation at the dendritic level (Ju et al. 2004; Liu 2004; Sutton et al. 2004), and there are now some indirect indications that HSP could act via a local mechanism ( $\mathrm{Ju}$ et al. 2004; Liu 2004; Sutton et al. 2004; Thiagarajan et al. 2005). However, as we have demonstrated (Figs. 2 and 3), new experiments that will alter dendritic activity differentially are necessary to determine whether the HSP mechanism is indeed local, global or perhaps both.

Theoretical studies on HSP have also limited themselves to "point-neuron" models (Toyoizumi et al. 2005; van Rossum et al. 2000; Yeung et al. 2004; but see (Siegel et al. 1994). These studies have all contributed important insights to the ongoing research on HSP. However, by definition, in a point neuron, there is no difference between $l$-HSP and $g$-HSP, and the essential notion of dendritic compartmentalization does not 
exist. Therefore we found it important to conduct our study using a detailed model of an electrically distributed dendritic tree.

As we have demonstrated, dendritic activity should not be regarded as strictly uniform, on the one hand, nor highly location specific, on the other hand. Instead, it seems to be distributed into individual compartments where "private" electrical interactions can take place. We have also introduced the idea that these compartments are linked together by "noncompartment" or "link" subregions that afford a more "public" venue for the travel and integration of activity. This can easily be seen by computing the cross-correlations between simultaneously recorded voltage traces from different dendritic sites and then clustering these cross-correlations into individual compartments (Fig. 4). This novel approach, can be used to delineate any type of dendritic compartmentalization [e.g., calcium compartments (Helmchen 1999; Zador and Koch 1994)], and unlike existing methods that are static and appropriate for passive dendrites (Koch et al. 1982; Zador et al. 1995), our approach is suitable for nonlinear excitable dendrites, taking into account the spatiotemporal patterns of synaptic input as well as possible changes to these patterns due to plasticity in synaptic strength and in intrinsic excitability. With the recent advances in imaging techniques and their improved spatiotemporal resolution (Antic et al. 2000; Iyer et al. 2006; Zochowski et al. 2000), this method could be applied for studying dendritic compartmentalization in real neurons. We further develop this issue in a separate study.

The HSP model we formulated is a voltage-driven one. This is justified by the fact that chronic changes in membrane potential are sufficient to elicit HSP (Burrone et al. 2002; Leslie et al. 2001; Paradis et al. 2001). Furthermore, at the large time scale, the average membrane potential contains a lot of information on dendritic activity (Koch et al. 1995) and thus could serve as a reasonable approximation for other measures of activity possibly driving HSP, such as intracellular calcium concentration (Thiagarajan et al. 2002; Yeung et al. 2004). Most importantly, our qualitative conclusions can be generalized to the relationship between HSP and dendritic compartmentalization for any HSP-controlled measure of activity (e.g., calcium concentration-driven HSP (Thiagarajan et al. 2002; Yeung et al. 2004) and calcium, rather than voltage, dendritic compartments (Helmchen 1999; Zador and Koch 1994)).

In conclusion, the interplay between dendritic compartmentalization and the mechanism underlying HSP has great import when considering the role of HSP in neuronal function. $g$-HSP, modifying synaptic weights together throughout the entire neuron, will completely overlook any compartmental organization, occasionally exacerbating deviated levels of activity within compartments. In contrast, $l$-HSP (although, in principle, equipped with the spatial resolution of a single synapse) will tend to act at the compartmental level (scaling synaptic weights together within each compartment), thus maintaining and regulating individual compartment activity. At the same time, whenever the overall activity in the cell is altered, requiring cell-wide adaptation, $l$-HSP can act as a global mechanism, changing all of the neuron's synapses together. Our assumption that $l$-HSP is driven by a uniform target membrane potential, $V_{\text {trg }}$, confers $l$-HSP with the additional advantage of leveling the average membrane potential throughout the dendritic tree, thus avoiding persisting axial current flow between different parts of the neuron and eliminating the excessive operation of ion pumps. The answer to the question whether HSP is local or global will have important implications to our understanding of the functioning of single neurons. Should they be considered distributed or centralized devices or could they perhaps alternate between these two modes?

\section{G R A N T S}

This work was supported by a grant donation from Mrs. Lily Safra ("Learning and Memory") and by National Institute of Mental Health Grant IR01-MH-59976-01A2, and Israeli Science Foundation Grant 10/256. I. Rabinowitch is supported by the Horowitz Fund Fellowship.

\section{REFEREN CES}

Abbott LF and Nelson SB. Synaptic plasticity: taming the beast. Nat Neurosci 3, Suppl: $1178-1183,2000$.

Abeles M. Corticonics: Neural Circuits of the Cerebral Cortex. Cambridge, UK: Cambridge Univ. Press, 1991.

Abraham WC and Bear MF. Metaplasticity: the plasticity of synaptic plasticity. Trends Neurosci 19: 126-130, 1996.

Antic S, Wuskell JP, Loew L, and Zecevic D. Functional profile of the giant metacerebral neuron of Helix aspersa: temporal and spatial dynamics of electrical activity in situ. J Physiol 527: 55-69, 2000.

Ariav G, Polsky A, and Schiller J. Submillisecond precision of the inputoutput transformation function mediated by fast sodium dendritic spikes in basal dendrites of CA1 pyramidal neurons. J Neurosci 23: 7750-7758, 2003.

Burrone J and Murthy VN. Synaptic gain control and homeostasis. Curr Opin Neurobiol 13: 560-567, 2003.

Burrone J, O'Byrne M, and Murthy VN. Multiple forms of synaptic plasticity triggered by selective suppression of activity in individual neurons. Nature 420: 414-418, 2002.

Desai NS, Cudmore RH, Nelson SB, and Turrigiano GG. Critical periods for experience-dependent synaptic scaling in visual cortex. Nat Neurosci 5: 783-789, 2002.

Frick A, Magee J, and Johnston D. LTP is accompanied by an enhanced local excitability of pyramidal neuron dendrites. Nat Neurosci 7: 126-135, 2004.

Goldberg J, Holthoff K, and Yuste R. A problem with Hebb and local spikes. Trends Neurosci 25: 433-435, 2002.

Golding NL, Kath WL, and Spruston N. Dichotomy of action-potential backpropagation in CA1 pyramidal neuron dendrites. J Neurophysiol 86: 2998-3010, 2001.

Golding NL and Spruston N. Dendritic sodium spikes are variable triggers of axonal action potentials in hippocampal CA1 pyramidal neurons. Neuron 21: 1189-1200, 1998.

Harms KJ, Tovar KR, and Craig AM. Synapse-specific regulation of AMPA receptor subunit composition by activity. J Neurosci 25: 6379-6388, 2005.

Hausser M and Mel B. Dendrites: bug or feature? Curr Opin Neurobiol 13: 372-383, 2003.

Helmchen F. Dendrites as biochemical compartments. In: Dendrites, edited by Stuart G, Spruston N and Hausser M. Oxford, UK: Oxford Univ. Press, 1999, p. $160-192$.

Iyer V, Hoogland TM, and Saggau P. Fast functional imaging of single neurons using random-access multiphoton (RAMP) microscopy. $J$ Neurophysiol 95: 535-545, 2006.

Johnston D, Hoffman DA, Colbert CM, and Magee JC. Regulation of back-propagating action potentials in hippocampal neurons. Curr Opin Neurobiol 9: 288-292, 1999.

Ju W, Morishita W, Tsui J, Gaietta G, Deerinck TJ, Adams SR, Garner CC, Tsien RY, Ellisman MH, and Malenka RC. Activity-dependent regulation of dendritic synthesis and trafficking of AMPA receptors. Nat Neurosci 7: 244-253, 2004.

Kilman V, van Rossum MC, and Turrigiano GG. Activity deprivation reduces miniature IPSC amplitude by decreasing the number of postsynaptic GABA(A) receptors clustered at neocortical synapses. J Neurosci 22: 1328-1337, 2002.

Koch C, Bernander O, and Douglas RJ. Do neurons have a voltage or a current threshold for action potential initiation? J Comput Neurosci 2: 63-82, 1995.

Koch C, Poggio T, and Torre V. Retinal ganglion cells: a functional interpretation of dendritic morphology. Philos Trans R Soc Lond B Biol Sci 298: 227-263, 1982. 
Larkum ME, Zhu JJ, and Sakmann B. Dendritic mechanisms underlying the coupling of the dendritic with the axonal action potential initiation zone of adult rat layer 5 pyramidal neurons. J Physiol 533: 447-466, 2001.

Leslie KR, Nelson SB, and Turrigiano GG. Postsynaptic depolarization scales quantal amplitude in cortical pyramidal neurons. J Neurosci 21: RC170, 2001

Lisman J and Spruston N. Postsynaptic depolarization requirements for LTP and LTD: a critique of spike timing-dependent plasticity. Nat Neurosci 8: $839-841,2005$

Liu G. Local structural balance and functional interaction of excitatory and inhibitory synapses in hippocampal dendrites. Nat Neurosci 7: 373-379, 2004.

Magee JC and Cook EP. Somatic EPSP amplitude is independent of synapse location in hippocampal pyramidal neurons. Nat Neurosci 3: 895-903, 2000.

Migliore M. Modeling the attenuation and failure of action potentials in the dendrites of hippocampal neurons. Biophys J 71: 2394-2403, 1996.

Migliore M, Hoffman DA, Magee JC, and Johnston D. Role of an A-type $\mathrm{K}^{+}$conductance in the back-propagation of action potentials in the dendrites of hippocampal pyramidal neurons. J Comput Neurosci 7: 5-15, 1999.

Moulder KL, Meeks JP, Shute AA, Hamilton CK, de Erausquin G, and Mennerick S. Plastic elimination of functional glutamate release sites by depolarization. Neuron 42: 423-435, 2004.

O'Brien RJ, Kamboj S, Ehlers MD, Rosen KR, Fischbach GD, and Huganir RL. Activity-dependent modulation of synaptic AMPA receptor accumulation. Neuron 21: 1067-1078, 1998.

Paradis S, Sweeney ST, and Davis GW. Homeostatic control of presynaptic release is triggered by postsynaptic membrane depolarization. Neuron 30: 737-749, 2001.

Poirazi P, Brannon T, and Mel BW. Arithmetic of subthreshold synaptic summation in a model CA1 pyramidal cell. Neuron 37: 977-987, 2003.

Polsky A, Mel BW, and Schiller J. Computational subunits in thin dendrites of pyramidal cells. Nat Neurosci 7: 621-627, 2004.

Rao A and Craig AM. Activity regulates the synaptic localization of the NMDA receptor in hippocampal neurons. Neuron 19: 801-812, 1997.

Rumsey CC and Abbott LF. Equalization of synaptic efficacy by activityand timing-dependent synaptic plasticity. J Neurophysiol 91: 2273-2280, 2004.
Siegel M, Marder E, and Abbott LF. Activity-dependent current distributions in model neurons. Proc Natl Acad Sci USA 91: 11308-11312, 1994.

Song S, Miller KD, and Abbott LF. Competitive Hebbian learning through spike-timing-dependent synaptic plasticity. Nat Neurosci 3: 919-926, 2000.

Sutton MA, Wall NR, Aakalu GN, and Schuman EM. Regulation of dendritic protein synthesis by miniature synaptic events. Science 304: 1979-1983, 2004.

Thiagarajan TC, Lindskog M, and Tsien RW. Adaptation to synaptic inactivity in hippocampal neurons. Neuron 47: 725-737, 2005.

Thiagarajan TC, Piedras-Renteria ES, and Tsien RW. alpha- and betaCaMKII. Inverse regulation by neuronal activity and opposing effects on synaptic strength. Neuron 36: 1103-1114, 2002.

Toyoizumi T, Pfister JP, Aihara K, and Gerstner W. Generalized Bienenstock-Cooper-Munro rule for spiking neurons that maximizes information transmission. Proc Natl Acad Sci USA 102: 5239-5244, 2005.

Turrigiano GG, Leslie KR, Desai NS, Rutherford LC, and Nelson SB. Activity-dependent scaling of quantal amplitude in neocortical neurons. Nature 391: 892-896, 1998.

Turrigiano GG and Nelson SB. Homeostatic plasticity in the developing nervous system. Nat Rev Neurosci 5: 97-107, 2004.

van Rossum MC, Bi GQ, and Turrigiano GG. Stable Hebbian learning from spike timing-dependent plasticity. J Neurosci 20: 8812-8821, 2000.

Wei DS, Mei YA, Bagal A, Kao JP, Thompson SM, and Tang CM. Compartmentalized and binary behavior of terminal dendrites in hippocampal pyramidal neurons. Science 293: 2272-2275, 2001.

Williams SR. Spatial compartmentalization and functional impact of conductance in pyramidal neurons. Nat Neurosci 7: 961-967, 2004.

Yeung LC, Shouval HZ, Blais BS, and Cooper LN. Synaptic homeostasis and input selectivity follow from a calcium-dependent plasticity model. Proc Natl Acad Sci USA 101: 14943-14948, 2004.

Zador A and Koch C. Linearized models of calcium dynamics: formal equivalence to the cable equation. J Neurosci 14: 4705-4715, 1994.

Zador AM, Agmon-Snir H, and Segev I. The morphoelectrotonic transform: a graphical approach to dendritic function. J Neurosci 15: 1669-1682, 1995.

Zochowski M, Wachowiak M, Falk CX, Cohen LB, Lam YW, Antic S, and Zecevic D. Imaging membrane potential with voltage-sensitive dyes. Biol Bull 198: 1-21, 2000. 